\title{
MBSE and MBSA with Capella and Safety Architect Tools
}

\author{
Marc Sango, Frédérique Vallée, Anne-Catherine Vié, \\ Jean-Luc Voirin, Xavier Leroux and Véronique Normand
}

\begin{abstract}
The development of critical systems is a challenging task that requires collaborative work for various purposes: specification, design and verification. Today, no single modeling language and environment covers all these aspects. ARCADIA and Capella ${ }^{\odot}$ are Model-based System Engineering (MBSE) method and tool developed for the system design process. ARCADIA/Capella also adopts a viewpoint-based description to describe engineering specialty, such as the safety engineering. Safety Architect $^{\circledR}$ is a MBSA (Model Based Safety Analysis) tool developed by ALL4TEC to analyze the robustness of design models. Indeed, Safety Architect can use design models imported from usual modelling tools, such as Capella, in order to perform classical safety analyses: automatic deduction of fault tree of the identified feared events. In this paper, we present our MBSE and MBSA approach developed in the Clarity project around Safety viewpoint in Capella and the import legacy into Safety Architect in order to realize safety analysis.
\end{abstract}

M. Sango (州) F. Vallée · A.-C. Vié

ALL4TEC, Changé, France

e-mail: Marc.Sango@all4tec.net

F. Vallée

e-mail: Frederique.Vallee@all4tec.net

A.-C. Vié

e-mail: Anne-Catherine.Vie@all4tec.net

J.-L. Voirin

Thales Mission Systems, Paris, France

e-mail: Jean-Luc.Voirin@ all4tec.net

$X$. Leroux

Thales Global Services, Paris, France

e-mail: Xavier.Leroux@all4tec.net

V. Normand

Thales Research and Technology, Paris, France

e-mail: Veronique.Normand@all4tec.net

G. Fanmuy et al. (eds.), Complex Systems Design \& Management,

DOI 10.1007/978-3-319-49103-5_22 